two control groups differed radically in their use of diuretics, and given that their model using control per case showed a significant odds ratio $>100$ associated with the use of diuretics, it may be important to determine potential synergies for risk mediated by hypokalaemia directly, as well as including the use of diuretics in regression analyses.

As several of the new, atypical antipsychotics recently have been shown to block the HERG $\mathrm{K}^{+}$channel, the clinical implications are that without a more complete understanding of the mechanism of risk, further studies examining this association for new atypical antipsychotic agents will require, where possible, prospective studies that can be used to determine the synergistic action of other known risk factors to be measured directly. Although mortality, even a low risk of mortality, is an unacceptable effect for a drug used to treat a non-fatal condition, the successful use of drugs such as thioridazine militates against the wholesale elimination of these drugs without due consideration for individual cases.

Choy, A. M., Lang, C. C., Chomsky, D. M., et al (1997) Normalization of acquired QT prolongation in humans by intravenous potassium. Circulation, 96, 2149-2154.

Davies, S. J., Cooke, L. B., Moore, A. G., et al (2002) Discontinuation of thioridazine in patients with learning disabilities: balancing cardiovascular atoxicity with adverse consequences of changing drugs. BMJ, 324 |519-152|.

Hancox, J. C. \& Witchel, H. J. (2000) Psychotropic drugs, HERG, and the heart (letter). Lancet, 356, 428

Keating, M.T. \& Sanguinetti, M. C. (200I) Molecular and cellular mechanisms of cardiac arrhythmias. Cell, 104, 569-580.

Lindquist, M. \& Edwards, I. R. (1997) Risks of nonsedating antihistamines (letter). Lancet, 349, 1322

Reilly, J. G., Ayis, S. A., Ferrier, I. N., et al (2002) Thioridazine and sudden unexplained death in psychiatric in-patients. British Journal of Psychiatry, 180, 515-522.

Roden, D. M. (1998) Taking the 'idio' out of

'idiosyncratic': predicting torsades de pointes. Pacing and Clinical Electrophysiology, 2I, 1029-1034.

H. J.Witchel, J. C. Hancox Cardiovascular Research Laboratories, Department of Physiology, School of Medical Sciences, University of Bristol, University Walk, Bristol BS8 ITD, UK

D. J. Nutt, S. Wilson Psychopharmacology Unit School of Medical Sciences, University of Bristol, UK

\section{Decision-making and euthanasia}

In a recent editorial Kelly \& McLoughlin (2002) highlight the fact that the uncertain prognosis of most psychiatric diseases means that the objective accuracy of decisions on 'physician-assisted suicide' and euthanasia in this category of patients cannot be certified.

One important psychological issue, which parallels these views but applies to all cases of physician-assisted suicide and active euthanasia, is that decisions on these issues may be influenced by unintentional and even unconscious biases. One example of this phenomenon was presented in a recent study in which Swedish jurors were presented with a case description of a severely brain-damaged patient who was taken out of a respirator in the presence of muscle-relaxing drugs. The jurors were, most likely out of concern for the patient, generally supportive of euthanasia. However, since we varied the gender of the patient, as presented in the case description, we were also able to see that both male and female jurors tended to be most supportive of this kind of euthanasia when it was administered to a patient who belonged to the opposite gender (Sjöberg \& Lindholm, 2003). Swedish jurors thus tended to be more impressed by the futility of the life of patients who were in important respects dissimilar to themselves.

Not only psychiatric, but almost all clinical decision-making is to a certain extent tentative and subject to the corrective forces of expectation and further empirical observations - but decisions that lead to the active and intentional termination of the life of a patient are not. We believe that this fact, which was also indirectly addressed by Kelly \& McLoughlin, is important not only to the discussion of whether physician-assisted suicide should be administered to psychiatric patients but also to the discussion of whether physicians should engage in euthanasia and physician-assisted suicide and whether psychiatrists should take the risk of sanctioning such activities by assessing the mental status of potential subjects of such interventions.

Kelly, B. D. \& McLoughlin, D. M. (2002) Euthanasia, assisted suicide and psychiatry: a Pandora's box. British Journal of Psychiatry, I8I, 278-279.

Sjöberg, R. L. \& Lindholm, T. (2003) Gender biases in decisions on euthanasia among Swedish jurors. Nordic Journal of Psychiatry, in press.

R. L. Sjöberg, T. Lindholm National Institute of Psychosocial Factors and Health, PO Box 220, 17177 Stockholm, Sweden

\section{More to social capital than Putnam}

I would like to comment on the editorial by McKenzie et al (2002) regarding social capital and mental health.

Putnam's conceptualisation of social capital is the one that has caught the interest of policy-makers in recent years but it is pre-dated, by at least a decade, by Bourdieu's $(1980,1985)$ theory of capital which, I would argue, has more relevance for the study of social and health inequalities. Portes $(1998,2000)$ gives an accessible account of this dynamic view of social capital.

One of Bourdieu's main insights is that people consciously participate to build their various forms of capital and then use them to their advantage. In this way, social capital is a property of the individual, acquired though it may be through group membership. More importantly, social capital (along with all the other forms of capital) is then implicated in the production and reproduction of the very inequalities it is generally thought to mediate against. This dialectic poses some very real questions for the study of health inequalities over the life course, especially with regard to the possibility of disentangling any direct effects of social capital on health from the indirect effects of social capital through increased social mobility and access to economic capital.

This dynamic view of social capital also allows health research to go beyond examining health 'status' to investigate its role in the onset of and recovery from illness and poor health. Those with low stocks of capital are more likely to become ill and take longer to recover or are less likely to recover at all. Further, they are more likely to suffer adverse consequences of their illness in other fields, such as regaining employment, thus contributing to the widening of health inequalities.

Although I agree with most of the editorial on the potential of social capital as a heuristic device in studies of mental health, I was disappointed that it gave the impression of theoretical or conceptual consensus on the issue. I hope that my brief sketch will encourage researchers to go further than Putnam's ideas.

Bourdieu, P. (1980) Le capital social: notes provisoires. Actes de la Recherche in Sciences Sociales, 3I, 2-3.

- (1985) The forms of social capital. In Handbook of Theory and Research for the Sociology of Education (ed. J. G. Richardson), pp. 24I-258. New York: Greenwood. 